\title{
Metal Effect on Reliability of Balise Transmission System Antennas
}

\author{
Linfu ZHU ${ }^{1}$, Kun LI, Junfei WANG and Yang LI \\ Standards \& Metrology Research Institute, China Academy of Railway Sciences \\ Corporation Limited, Beijing 100081, China
}

\begin{abstract}
In railway networks, metal objects near the balise transmission system can affect the performance of antennas in the system operating at $27.095 \mathrm{MHz}$ and 4.23 MHz. In this paper, the performance of the antennas in the proximity of steel rails and metal plates are simulated and tested in terms of resonant frequency, magnetic field distribution. The results show that the metal effect is dependent on the distance between the metal and the antenna. They shift the resonant frequency and change the magnetic field distribution. In order to solve the problem, we find that the antennas should keep specified distance with the steel rails. Also, a metal plate is fixed on the back of on-board antenna unit to reduce the sensitivity to metal. These methods increase the robustness and reliability of balise transmission system antennas in railway signalling.
\end{abstract}

Keywords. Railway signal, train control system, balise

\section{Introduction}

In 1964, the first high-speed train in the world started its commercial operation on the Tokaido Shinkansen in Japan. In 2008, China opened the first high-speed railway. Till now, high-speed railway has changed the travelling style of passengers for its safe, convenient, fast, punctual, comfortable and human-based travelling experience. In China, the high-speed railway has been a means of public transport. There are more than 1.3 billion passengers who take high-speed railway for travelling every year. Since high-speed railways are safety-critical systems, the Train Operation Control System is particularly adopted to guarantee the operation safety.

The location accuracy in Train Operation Control System is highly critical for the train operation safety as the permitted speed depends on the position of each train. Balise is an electromagnetic transponder installed between rails that supplies accurate and precise location information to the vital computer of the Train Operation Control System. The accumulated location inaccuracies over distance are corrected when the train passes a balise [1-3]. It also transmits the safety-related line information from wayside to the on-board vital computer [4]. Hence, it is treated as an essential component for European Train Control Systems and Chinese Train Control Systems in high-speed railways. The failure to receive the telegrams of two successive balise groups requires stopping the train [5]. Therefore, the reliability of Balise Transmission

\footnotetext{
${ }^{1}$ Corresponding author: Linfu Zhu, email:zhulinfu2008@163.com。
} 
System is critical for the Train Operation Control System in high-speed railways and metro lines.

The balise transmission system is made up by balises installed on railway tracks and on-board Balise Transmission Module (BTM) [6,7].

China has the longest high-speed railway route in the world. The mileage has reached 35,000 kilometers. Topically, one balise group that include 3 balises which is installed every 2 kilometers in Chinese high-speed railways. So the total number of balises is extremely huge. On the other hand, the 35,000 kilometers high-speed railway travels through almost every type of climates and environments in China. The balise transmission system is an outdoor equipment, so it will deal with an extreme climate and harsh environment, including the rainstorm, strong wind, extremely cold and desert. The surface of the balise would be covered by environmental mediums, such as rain, sand, dust, ice and snow. The balise in the freight railway line would be covered by fallen iron ore, iron dust, coal dust, oil and grease. Meanwhile, the surrounding of the balise and on board antenna is full of all kinds metal objects, including the train body, the steel rails, the metal mounting bracket, and the fallen metal on the railway $[8,9]$. In addition, there are several mounting base, such as the concrete roadbed, concrete sleeper and wood sleeper. The metal is lossy object for the electromagnetic wave. Compared with the nominal air condition, the dynamic contact length and number of non-overlapping good telegrams of the balise transmission system are decreased with the debris, such as metal and ice [10].

The balise transmission system is a special application of Radio Frequency Identification (RFID) in railway signalling systems. The surrounding environment would affect the RFID system. In order to meet the boundary conditions, the metals or dielectrics (such as water) decrease electric and magnetic field, shift the resonant frequency and change the antenna impedance matching [11-13]. The proximity effects are strongly dependent on the metal plate size, distance between the plate and the antenna as well as the plate orientation $[12,14]$. When the antenna is positioned within less than $3 \mathrm{~cm}$ from a metal surface, the resonant frequency increases from $13.56 \mathrm{MHz}$ to $14.75 \mathrm{MHz}$. The wider angle between the antenna and the metallic surface introduces the less shift of the resonant frequency [15].

The effective read range of an RFID system depends on environmental effects [16]. The dielectric materials reduce the read range of the tag [17]. A planar dipole-based antenna matched with a small inductive coil is mounted on different mounting materials. The maximum range is eight meters for materials with a low permittivity, and the maximum range is close to two meters for denser materials or materials with a higher permittivity [18].

There are several methods to reduce the medium and metal effect on the RFID antenna. The antenna is detuned when it is in the vicinity of metals and liquids or kept a distance away from the metal frame [19]. A permanent metal plate is fixed at the back of the loop antenna. In order to reduce the currents induced in the conducting bodies in the proximity of the antenna, flexible polymer-ceramic composite materials with high permittivity are adopted as substrates for single-layer antennas in metal-mountable tags. They increase the antenna metal separation [20]. The generic algorithm is adopted to integrate the complexity of real RFID environment into the design methodology, which is mandatory for robustness property [21]. To compensate for the changed inductance of the RFID antenna due to the metal tube and the inductance disturbance coming from the liquid in the extreme downhole environments, an adaptive antenna tuning unit with a variable capacitor array is presented to improve the system's robustness and decrease 
the sensitivity in volatile application conditions [22]. Considering the special application environment of the balise transmission system, detuning, keeping a distance with mediums or metal and fixing a metal plate are researched in this paper.

The loop antennas of balise transmission system is modelled and studied in perfect conditions (free air space conditions) [6,7]. However, imperfect conditions, such as the dielectric and metal near the system would decrease the effective communication distance. The solution to the potential problem in perfect conditions is to research the metal effect on these antennas. The metal would change the magnetic field distribution and the resonant frequency of the antennas[23-24]. In this paper, steel rails and metal plates, are selected to study.

\section{Modelling and Research Configuration}

Figure 1(a) shows the balise transmission system antennas in the reference coordinate, which includes on-board antenna unit and the balise. The on-board antenna unit includes $27.095 \mathrm{MHz}$ transmitting antenna and $4.23 \mathrm{MHz}$ receiving antenna, which is mounted below the train body. The balise includes $27.095 \mathrm{MHz}$ receiving antenna and 4.23 MHz transmitting antenna, which is mounted between the two rails. Thus, there are totally four antennas in balise transmission system. The distance between the balise and on-board antenna unit ranges from $220 \mathrm{~mm}$ to $460 \mathrm{~mm}$ along Z-axis.

The on-board $27.095 \mathrm{MHz}$ transmitting antenna radiates tele-powering signal continually. The operation frequency is $27.095 \mathrm{MHz}$. When the train passes the balise, the $27.095 \mathrm{MHz}$ receiving antenna in the balise receives the magnetic flux from the onboard 27.095 MHz transmitting antenna and induces the current, which is taken as the power for balise operation. Then the balise is activated. The $4.23 \mathrm{MHz}$ transmitting antenna in balise starts to transmit FSK uplink signal to the on-board antenna unit. The 4.23 $\mathrm{MHz}$ receiving antenna in the on-board antenna unit induces the uplink signal. The induced signal is demodulated and decoded into telegram. At last, the telegram is sent to the on-board vital computer to calculate the permitted speed line and control the real operation speed. When the train is far away from the balise, it stops transmitting uplink signal and goes in dormancy status.

Figure 1(b) illustrates the reference axes. The $\mathrm{X}$-axis is parallel with the rails and the positive direction is same with train travel direction; the $\mathrm{Y}$-axis is perpendicular to the rails and level with the top of rails; the Z-axis directs upwards and has right angles to the rail plane. The origin of the coordinates is in the rail bottom plane and in the middle of the track.

The copper wire loop with the matching and tuning circuit is shown in Figure 1(c), whose relative permeability is 1 and electric conductivity is $5.8 \times 10^{\wedge} 7 \mathrm{~S} / \mathrm{m}$. The thickness of copper wire is $0.072 \mathrm{~mm}$. The four antennas have same dimension. The substrate material is FR4, and its dimension is $430 \mathrm{~mm} \times 240 \mathrm{~mm} \times 2 \mathrm{~mm}$.

For the $27.095 \mathrm{MHz}$ transmitting and receiving antennas, the $\mathrm{Cs}$ is $15.1 \mathrm{pF}$; the $\mathrm{Cp}$ is $11.8 \mathrm{pF}$; the Rs is $15 \Omega$. For the $4.23 \mathrm{MHz}$ transmitting and receiving antennas, the Cs is $662 \mathrm{pF}$; the $\mathrm{Cp}$ is $784 \mathrm{pF}$; the $\mathrm{Rs}$ is $7.65 \Omega$.

The equivalent circuit of the loop antenna with matching and tuning circuit is illustrated in Figure 1(d). The matching and tuning circuit is same with that in the Figure 1(c). $R$ represents the equivalent serial resistance of the loop antenna. $L$ represents the equivalent serial inductance of the loop antenna. $C_{s}$ is the serial tuning 
capacitance and $C_{p}$ is the parallel tuning capacitance. $R_{s}$ is the serial matching resistance. The reactance of the loop antenna with matching and tuning circuit is derived as

$$
X=\frac{1}{j \omega C_{s}}+\frac{j \omega L}{1-\omega^{2} C_{p} L}
$$

When the reactance $\mathrm{X}$ is zero, the loop antenna is tuned. The turned frequency $\mathrm{f}$ is derived as

$$
f=\frac{1}{2 \pi} \sqrt{\frac{1}{L\left(C_{s}+C_{p}\right)}}
$$

The environmental mediums and metal surrounded the loop antenna will change the magnetic flux through the antenna as different permittivity and permeability. The definition of inductance is

$$
L=\frac{\psi}{I}
$$

Where $\psi$ is the magnetic flux generated by current $I$. From expression (2) and (3), the variation of magnetic flux $\psi$ will change the loop inductance $L$, which shifts the tuned frequency $f$.

$27.095 \mathrm{MHz}$

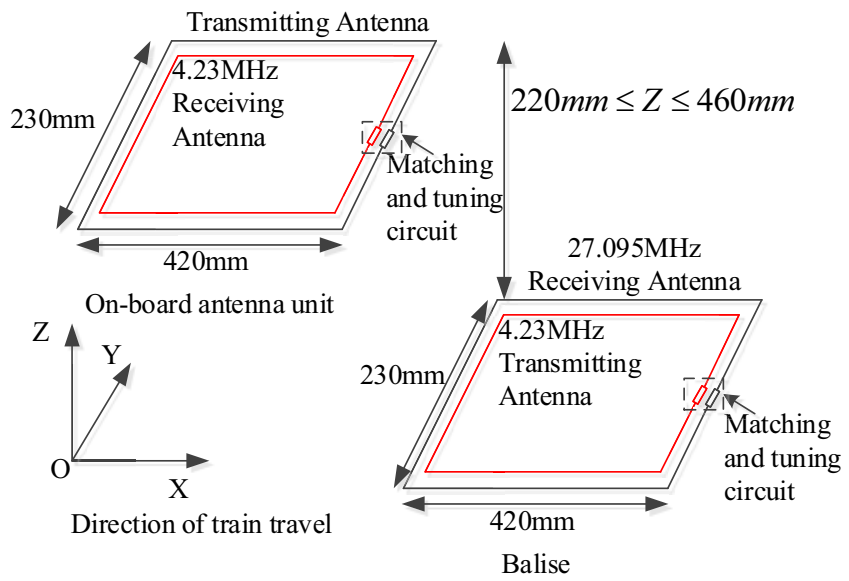

(a) Balise transmission system antennas 


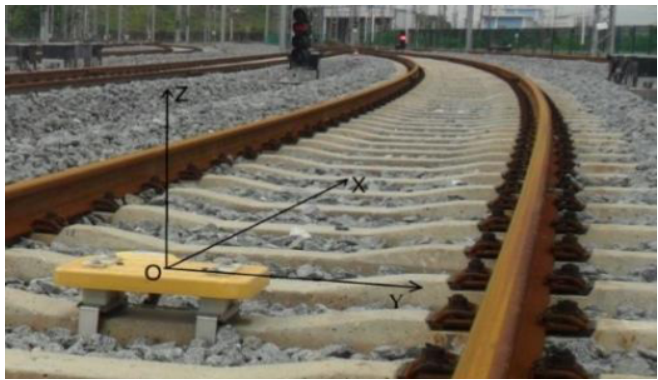

(b) Reference axes

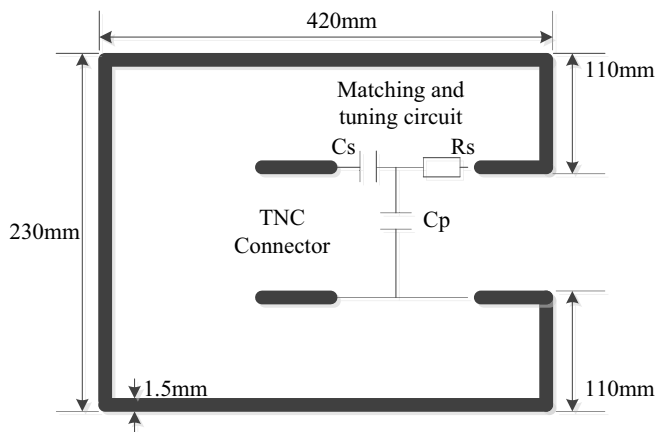

(c) Loop antenna with matching and tuning circuit

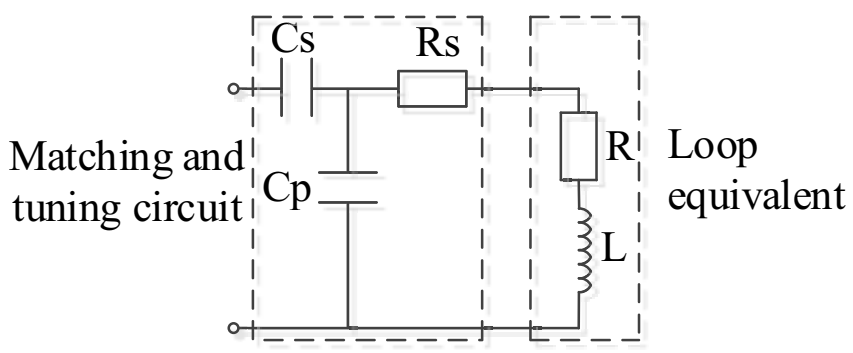

(d) Equivalent circuit of the loop antenna

Figure 1. Balise transmission system antennas and reference axes

\section{Metal Effect Analysis}

The balise transmission system includes the wayside balise, the onboard antenna unit and the onboard BTM. The metal would affect the system antennas in the wayside balise and the onboard antenna. As the distance between the wayside and the onboard antenna is larger than the metal effect distance for the near field magnetic antenna, the steel rail generates little effect to the onboard antenna unit. Meanwhile, the onboard metal generates little effect to the wayside balise. 


\subsection{Steel Rail}

The balise is mounted between two steel rails. The distance between balise and rails will influence the performance of balise. Two steel rails are included in the model and simulation is repeated to investigate the magnetic field distribution.

For the steel rails, its relative permeability is 1 and electric conductivity is $7.69 \times 10^{\wedge} 6 \mathrm{~S} / \mathrm{m}$. The distance between two rails is $1067 \mathrm{~mm}$. The Y-axis coordinate in the middle of the two rails is 0 . The balise is moved in the range $(-400 \mathrm{~mm}, 400 \mathrm{~mm})$ along the Y-axis, which is shown in Figure 2. The black rectangle in the middle of the two rails represents the two loop antennas in the balise, and the red circle is the feeding point.

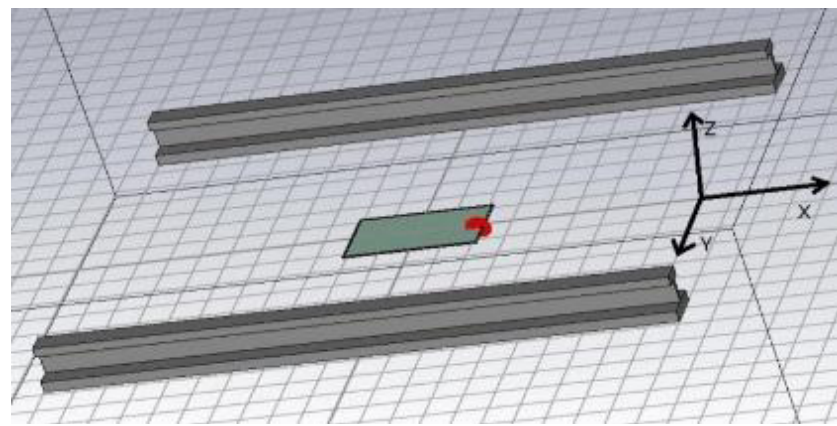

Figure 2. Balise with two steel rails separated by $1067 \mathrm{~mm}$

The base of rails is earth. In order to reduce the earth attenuation, the foam is between the antennas and the earth base, whose thickness is $4 \mathrm{~mm}$.

The two steel rails would change the magnetic field distribution. The electrical center of the $27.095 \mathrm{MHz}$ transmitting antenna is located in the point $(0,0,460 \mathrm{~mm})$. The amplitude of the excitation is $8.94 \mathrm{~V}$ and the power is $40 \mathrm{~W}$. It is assumed that the balise is mounted on the plane of $Z=100 \mathrm{~mm}$. The magnetic field distributions of the received tele-powering on the XOY plane with $Z=100 \mathrm{~mm}$ with and without rails are shown in Figure 3(a,b) respectively. In Figure 3(a), the two black bars represent the two rails. It indicates that the two rails blocks the magnetic field propagation along Yaxis, which is beneficial for the crosstalk reduction of two close railway lines. The maximum magnetic field is in the projection of $27.095 \mathrm{MHz}$ transmitting antenna in the XOY plane with $Z=100 \mathrm{~mm}$. So the balise and the on-board antenna unit should have the same $\mathrm{Y}$-axis coordinate to get the maximum tele-powering.

For the communication of the balise transmission system, the effective magnetic field is the Z-axis component. With the same simulation setup, Figure 4(a-c) show the $\mathrm{Z}$-axis component of the tele-powering magnetic field along $\mathrm{X}$-axis, $\mathrm{Y}$-axis and $\mathrm{Z}$-axis respectively. The results have been converted to $\mathrm{dB}$ relative to $1 \mathrm{~A} / \mathrm{m}$. These three figures indicate that the rails reduce the magnetic field. In Figure 4(b), the part of the side lobe closest to the main lobe with rail is much lower than that without rail. The Yaxis has two crossings with the two rails. As the above analysis of Figure 3(a), the magnetic field near the crossings is damped or reflected. Figure 4(c) indicates that the field is symmetrical. But only the left part is effective for communication, which is from $\mathrm{Z}=0 \mathrm{~mm}$ to $\mathrm{Z}=460 \mathrm{~mm}$. The right part is noise for other on-board devices. 


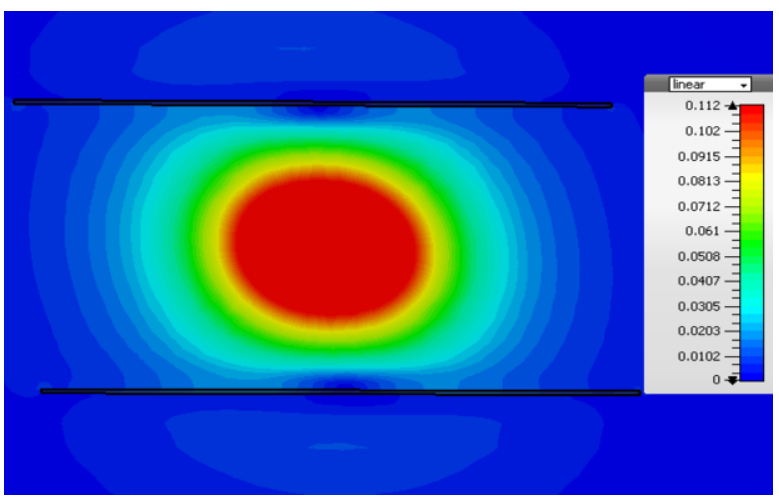

(a) With two steel rails

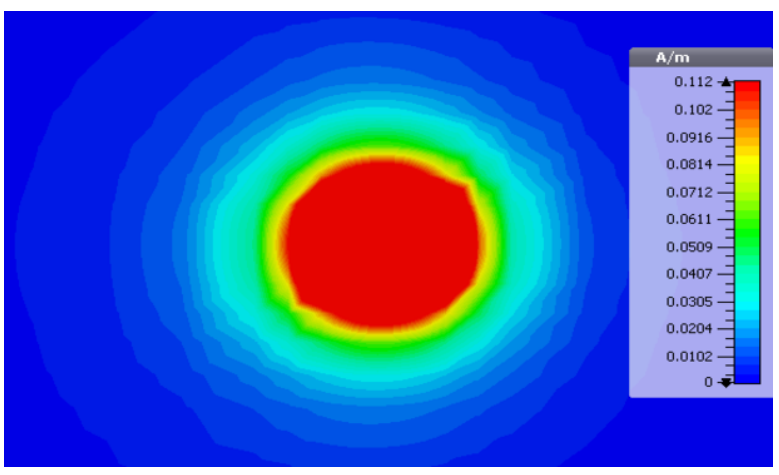

(b) Without two steel rails

Figure 3. Magnetic field distribution
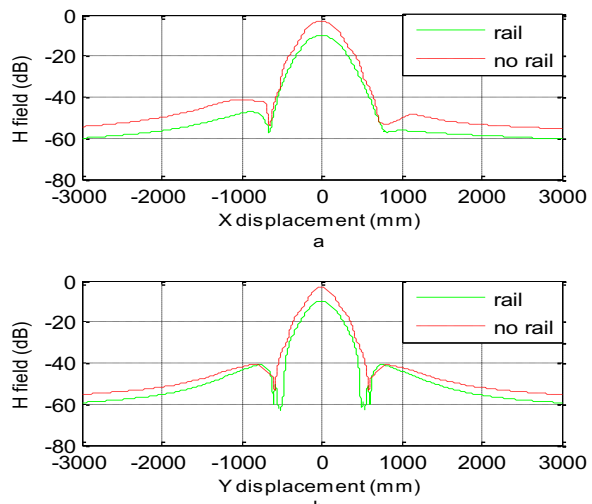

b

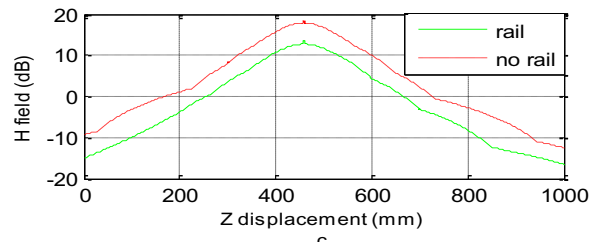

Figure 4. The $\mathrm{Z}$-axis component of the tele-powering magnetic field 


\subsection{Metal Plate}

The train body is a huge metal for the on-board antenna unit. In order to test the metal effect on the on-board antenna unit, one steel plate is approached in parallel to the 27.095 MHz transmitting antenna and 4.23 MHz receiving antenna respectively. The steel plate dimension is $450 \mathrm{~mm} \times 400 \mathrm{~mm} \times 1 \mathrm{~mm}$. The distance between the steel plate and both antennas is varied from $210 \mathrm{~mm}$ to $10 \mathrm{~mm}$.

The resonant frequency and return loss S11 of both antennas in the on-board antenna unit are shown in the Figure 5(a,b). The two figures indicate that a closer distance between the on-board antenna unit and the steel plate results in a greater frequency shift. For the $27.095 \mathrm{MHz}$ transmitting antenna, the measured resonant frequency of $26.99 \mathrm{MHz}$ when the distance is $210 \mathrm{~mm}$ is shifted to $30.42 \mathrm{MHz}$ when the distance is $10 \mathrm{~mm}$.

Figure 5(b) illustrates that the $27.095 \mathrm{MHz}$ transmitting antenna has the highest resonant frequency (31.79 $\mathrm{MHz}$ for simulation and $30.42 \mathrm{MHz}$ for measurement) when the distance between the steel plate and the antenna is $10 \mathrm{~mm}$. When the distance is larger than $110 \mathrm{~mm}$, the resonant frequency almost does not change (26.94 MHz for simulation, $26.99 \mathrm{MHz}$ for measurement).

In order to reduce the metal sensitivity and increase the robustness of the on-board antenna unit, a metal plate $(450 \mathrm{~mm} \times 400 \mathrm{~mm} \times 1 \mathrm{~mm})$ is fixed on the back of on-board antenna unit with a distance $30 \mathrm{~mm}$. The $27.095 \mathrm{MHz}$ transmitting antenna and 4.23 $\mathrm{MHz}$ receiving antenna are retuned at $27.095 \mathrm{MHz}$ and $4.23 \mathrm{MHz}$ respectively. The train body is simulated by the second metal plate $(450 \mathrm{~mm} \times 400 \mathrm{~mm} \times 1 \mathrm{~mm})$. Then second steel plate is approaching to the side where the first steel plate is. The both steel plates are parallel with both loop antennas in the on-board antenna unit.
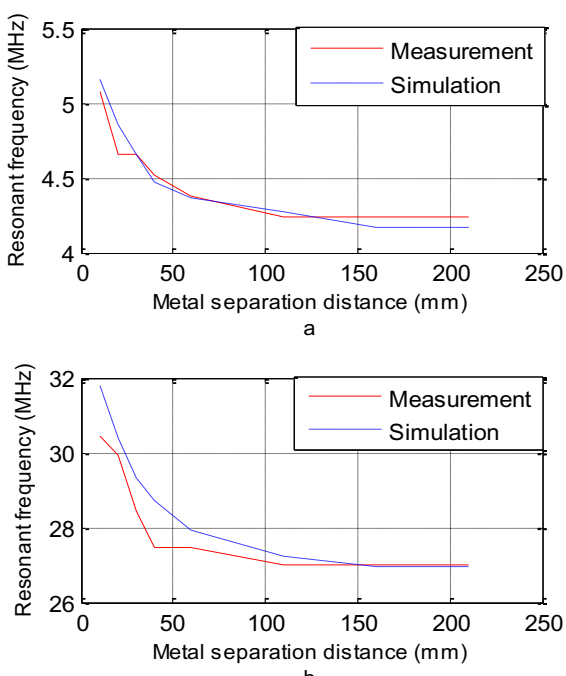

Figure 5. S11 with one metal plate for $4.23 \mathrm{MHz}$ receiving antenna and $27.095 \mathrm{MHz}$ transmitting antenna 

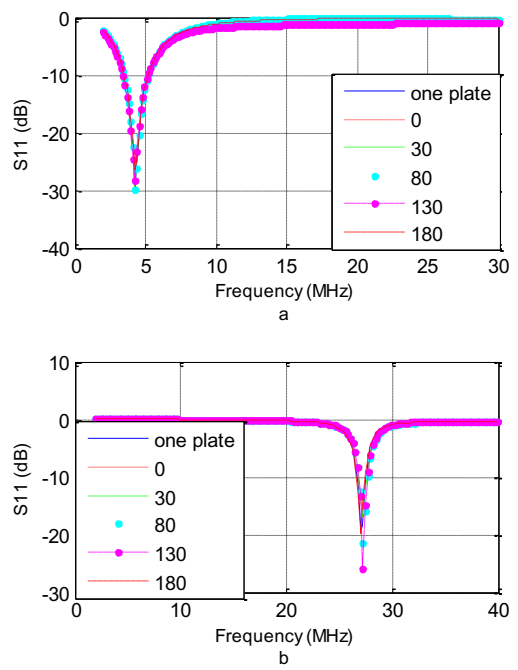

Figure 6. Measured $\mathrm{S} 11$ with two metal plates for $4.23 \mathrm{MHz}$ receiving antenna and $27.095 \mathrm{MHz}$ transmitting antenna

The measured S11 of both antennas with two metal plates are shown in the Figure $6(\mathrm{a}, \mathrm{b})$. The measured resonant frequency shift of $27.095 \mathrm{MHz}$ transmitting antenna is $0.24 \mathrm{MHz}$ with the distance between two metal plates in the range $(30 \mathrm{~mm}, 180 \mathrm{~mm})$. When the distance is $0 \mathrm{~mm}$, the resonant frequency with two plates is equal to that with one plate. For the $4.23 \mathrm{MHz}$ receiving antenna, all the resonant frequencies with two metal plates are same which are $4.24 \mathrm{MHz}$. The results indicate that the second metal plate has little effect on the both antennas in the on-board antenna unit. The fixed metal plate and the train body should be as close as possible.

The fixed metal plate on the back of the on-board antenna unit also change the magnetic field distribution. As analysis of Figure 4(c), the magnetic field above $Z=460$ $\mathrm{mm}$ plane is interference for other devices. On the basis of the simulation setup which generates the magnetic field distribution as Figure 4(c), the first steel plate is fixed on the back of the $27.095 \mathrm{MHz}$ transmitting antenna. The Z-axis component of the telepowering magnetic field along $Z$-axis with $X=0 \mathrm{~mm}$ and $Y=0 \mathrm{~mm}$ is shown in Figure 7 . Compared with no metal plate, the magnetic field with one metal plate above $Z=460$ $\mathrm{mm}$ plane is reduced, which improves its electromagnetic compatibility performance. But it reduces the effective signal between $Z=0 \mathrm{~mm}$ to $Z=460 \mathrm{~mm}$, which will decrease the effective communication distance.

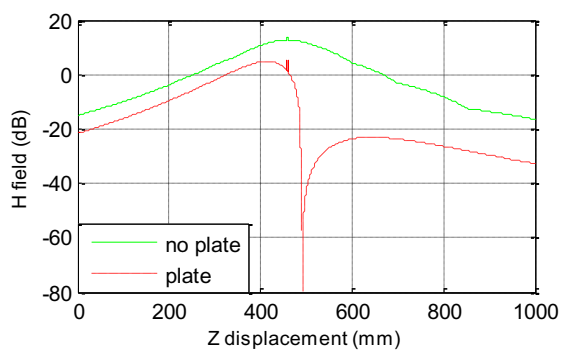

Figure 7. Z-axis component of the tele-powering magnetic field along $\mathrm{Z}$-axis $(\mathrm{X}=0 \mathrm{~mm}, \mathrm{Y}=0 \mathrm{~mm})$ with one metal plate 


\section{Conclusion}

In this paper, we study the antenna performance of a balise system in high-speed railways under real-world application environment. In specific, a variety of practical cases that involve resonant frequency, and field distribution in the vicinity of steel rails and metal plate are explicitly considered. Through a series of simulations and experiments, the following conclusions are obtained:

(1) The two rails would reduce the magnetic field and shift the resonant frequency of balise $27.095 \mathrm{MHz}$ receiving antenna and alter the field distribution.

(2) One metal plate is fixed on the back of the on-board antenna unit to solve the detuning problem after installing. The distance between the plate and the train body should be as close as possible. It increases the robustness and decreases the sensitivity to metal. Meanwhile, the electromagnetic compatibility performance of the on-board antenna unit is improved.

These above methods can be applied and adopted in practical applications of balise systems for high-speed railways that could enhance the reliability, robustness and safety level of train operation systems.

\section{Acknowledgement}

Research is supported by China Railway Scientific and Technology Research Program Foundation (Number: J2019G009) and the Foundation of Standards \& Metrology Research Institute, China Academy of Railway Sciences (Number:BJ2019S17).

\section{References}

[1] Farooq J, Soler J. Radio communication for Communications-Based Train Control (CBTC): A tutorial and survey. IEEE Communications Surveys \& Tutorials, 2017; (99):1-26.

[2] Chen DW, Wang LJ, Li LX. Position computation models for high-speed train based on support vector machine approach. Applied Soft Computing, 2015;30:758-766.

[3] Yin JT, Chen DW, Tang T, Zhu LF, Zhu W. Balise arrangement optimization for train station parking via expert knowledge and genetic algorithm. Applied Mathematical Modelling, 2016;40 (19):85138529.

[4] Lundberg P. Eurobalise Transmission System: A technical overview, Advancement of the Science of Railway Signalling Proceedings 2002/2003': 24-30.

[5] Dhahbi S, Abbas-Turki A, Hayat S, El Moudni A. Study of the high-speed trains positioning system: European signaling system ERTMS / ETCS', 4th International Conference on Logistics, 2011;468-473.

[6] Zhao LH, Jiang Y. Modeling and optimization research for dynamic transmission process of balise telepowering signal in high-speed railways. Progress in Electromagnetics Research, 2013; 140: 563-588.

[7] Zhao LH, Jiang Y. Modeling and simulation of balise up-link data transmission based on finite element method. Journal of Theoretical \& Applied Information Technology.2012; 46 (2): 867-874.

[8] Union Industry of Signalling. UNISIG SUBSET-036: Form Fit Function Interface Specification for Eurobalise. Brussels: UNISIG Consortium, 2012.

[9] Sancho JI, Mendizabal J, Gurutzeaga I, Villaro A, Adin I, Bistue G. HF antenna design optimization for railway spot signalling systems. Microwave and Optical Technology Letters. 2011;53 (12):2742-2746.

[10] Zhao HB, Sun SP, Li W. Dynamic characteristics modelling and adaptability research of the balise transmission module in high speed railways. WIT Transactions on the Built Environment. 2010, 114: 475-482.

[11] Griffin JD, Durgin GD, Haldi A, Kippelen B. Radio link budgets for $915 \mathrm{MHz}$ RFID antennas placed on various objects. Texas Wireless Symposium. 2005: 22-26. 
[12] Qing XM, Chen ZN. Proximity effects of metallic environments on high frequency RFID reader antenna: study and applications. IEEE Transactions on Antennas and Propagation. 2007;55 (11): 3105 3111.

[13] Xianming Qing XM, Goh CK and Chen ZN. A broadband UHF near-Field RFID antenna. IEEE Transactions on Antennas and Propagation. 2010; 58(12):3829-3838.

[14] M. Periyasamy, R. Dhanasekaran. Assessment and analysis of performance of $13.56 \mathrm{MHz}$ passive RFID in metal and liquid environment. 2014 International Conference on Communications and Signal Processing (ICCSP). 2014: 1122-1125.

[15] Ilie Finis, Valentin Popa, Alexandru Lavric, Codrin Males, Stefan Sfichi. Performance evaluation of 13.56 MHz RFID antenna operating in metallic environments. 2012 20th Telecommunications Forum. 2012: 1210-1213.

[16] Moghaddam SS. Modeling the environmental effects on the radiated fields of a passive RFID system. International Journal of Applied Electromagnetics and Mechanics. 2013; 42 (4): 539-559.

[17] Basat SS, Lim K, Laskar J, Tentzeris MM. Design and modeling of embedded 13.56 MHz RFID antennas. IEEE Antennas and Propagation Society International Symposium. 2005: 64-67.

[18] T. Deleruyelle, P. Pannier, M. Egels, E. Bergeret. An RFID tag antenna tolerant to mounting on materials. IEEE Antennas and Propagation Magazine. 2010; 52 (4): 14-19.

[19] Dobkin DM, Weigand SM. Environmental effects on RFID tag antennas. Microwave Symposium Digest. 2005: 135-138.

[20] Björninen T, Sydänheimo L, Ukkonen L, Rahmat-Samii Y. Advances in antenna designs for UHF RFID tags mountable on conductive items. IEEE Antennas and Propagation Magazine. 2014; 56 (1):79103.

[21] Chaabane H, Perret E, Tedjini S. A methodology for the design of frequency and environment robust UHF RFID tags. IEEE Transactions on Antennas and Propagation. 2011;59 (9): 3436-3441.

[22] Zhu JX, Tao B, Yin ZP. A customized RFID-Based sensor system for intelligent oilwell. IEEE Sensors Journal. 2016;16 (13): 5426-5432.

[23] Xu N. Study on the Performance of Balise Transmission Module Antenna after Adding Shielding Plate. China Railway Science. 2017; 38(3): 110-115.

[24] Bansal R. Near field magnetic communication. IEEE Antennas and Propagation Magazine. 2004; 46 (2): 114-115. 